\title{
REDISTRIBUIÇÃO ENTRE FRAÇÕES E TEORES DISPONÍVEIS DE ZINCO EM SOLOS INCUBADOS COM LODO DE ESGOTO(1)
}

\author{
J osângela do Carmo Trezena de Araújo(2) \& Clístenes \\ Williams Araújo do Nascimento(3)
}

\begin{abstract}
RESUMO
A utilização agrícola de lodo de esgoto é uma da melhores opções para a disposição final deste resíduo. Contudo, em virtude da presença de metais pesados no lodo, torna-se importante a determinação da disponibilidade e das formas nas quais estes metais se encontram em solos tratados com esse resíduo. Este trabalho objetivou estudar a redistribuição entre frações e os teores disponíveis de $\mathrm{Zn}$ em solos incubados com lodo de esgoto. Foram utilizadas amostras de dois solos com diferentes características químicas e físicas às quais foram adicionadas cinco doses de lodo de esgoto (equivalentes a 0; 40,5; 81; 162 e $243 \mathrm{Mg} \mathrm{ha}^{-1}$ ). Nos períodos de incubação de 30, 60, 90, 120, 150 e 180 dias, foram coletadas amostras para determinação do Zn disponível, extraído por DTPA, e da sua distri bui ção entre frações do solo. Os resultados mostraram que a adição de doses crescentes de lodo de esgoto aumentaram os teores de $\mathrm{Zn}$ nos solos extraídos por DTPA; entretanto, de maneira geral, estes teores não aumentaram com o decorrer do tempo de incubação. Houve di minuição da mobilidade de Zn nos solos incubados com lodo, com transferência do elemento ligado à fração matéria orgânica, em sua maior parte, para a fração residual. Para as condições estudadas, a máxima dose de Zn a ser fornecida por aplicação de lodo de esgoto pela legislação americana não promoveu teores fitotóxicos de Zn no solo.
\end{abstract}

Termos de indexação: fracionamento, mobilidade de Zn, biossólido.

\footnotetext{
(1) Parte da Tese de Mestrado do primeiro autor, apresentada ao PPGCS, Universidade Federal Rural de Pernambuco - UFRPE. Recebido para publicação em abril de 2004 e aprovado em maio de 2005.

(2) Aluna de Pós-Graduação, PPGCS, Universidade Federal Rural de Pernambuco - UFRPE. CEP 52171-900 Recife (PE). E-mail: josangela@truenet.com.br

(3) Professor do Departamento de Agronomia, UFRPE. Bolsista do CNPq. E-mail: cwanascimento@yahoo.com
} 


\title{
SUMMARY: ZINC REDISTRIBUTION AMONG SOIL FRACTIONS AND CONCENTRATION OF AVAILABLE ZN IN SEWAGE SLUDGE- TREATED SOILS
}

\begin{abstract}
One of the best options for final disposal of sewage sludge is its use for agricultural purposes. Excessive heavy metal concentrations in sludge, however, pose a risk to the environment. It is ther fore important to determine not only the availability, but also the distribution of such metals in different soil fractions as a result of sludgeapplication. Our study ai med at evaluating both the redistribution of $\mathrm{Zn}$ among soil fractions and the $\mathrm{Zn}$ DTPA available concentration in sludgeincubated soils. Samples of two contrasting soils were used in the experiment in combination with five doses of sewage sludge $(0 ; 40.5 ; 81$; 162; and $\left.243 \mathrm{Mg} \mathrm{ha}^{-1}\right)$. Soil samples were collected to eval uate the available $\mathrm{Zn}$ content extracted by DTPA and the distribution in soil fractions after six periods of incubation (30, $60,90,120,150$, and 180 days). Results showed that sludge application increased the DTPA-extracted availableZn content, al though theavailableZn content showd no increase as a function of the incubation period. Zn mobility decreased in thesludge-incubated soils since the organically bound $\mathrm{Zn}$ was mainly redistributed to residual fractions. For the conditions of this study, the maximal annual loading rate of $Z n$ through sewage sludge suggested by theUSEPA (U nited States, 1993) did not lead to phytotoxic Zn concentrations in soils.
\end{abstract}

Index terms: fractionation, Zn availability, biosolid.

\section{INTRODUÇÃO}

Em vários países do mundo, a utilização de lodo de esgoto na agricultura tem-se tornado prática comum em virtude de sua potencialidade em aumentar a fertilidade do solo e promover mel horias nas qualidades físicas e químicas, atuando como condicionador de solos. No entanto, apesar desses efeitos benéficos, este resíduo pode conter metais pesados em teores relativamente elevados, o que pode resultar em contaminação de solos.

Diversos trabal hos no Brasil tem-se preocupado coma avaliação dos possíveis problemas ambientais relacionados com a utilização agrícola do lodo de esgoto, principal mente com a introdução de metais pesados no sistema solo (Anjos \& Mattiazzo, 2000; Oliveira \& Mattiazzo, 2001; Nascimento et al., 2004), embora pouca atenção tenha sido dada à avaliação das transformações dos metais no solo em relação ao tempo de contato sol o-lodo de esgoto. Em um dos poucos trabalhos em que essa avaliação foi feita (Oliveira et al., 2003), utilizou-se lodo enriquecido com metais. Entretanto, de acordo com Parveen et al. (1994), podeser mais realístico estudar os metais natural mente presentes no lodo e sua redistribuição nos solos.

Dentre os vários metais presentes no lodo de esgoto da Companhia Pernambucana de Saneamento (COMPESA), o Zn é particularmente importante pela sua alta concentração, disponibilidade para planta e potencial de ecotoxidez (Nascimento et al., 2004), razão pela qual se torna importante o monitoramento das transformações temporais deste el emento em solos tratados com lodo de esgoto.
Como explica Han et al. (2003), a redistribuição de metais em solos écaracterizada por uma retenção inicial rápida seguida de reações lentas, dependendo da espécie do metal, das propriedades do solo, do teor de metal e do tempo de incubação, passando de formas mais lábeis para mais estáveis deacordo com o tempo. Neste sentido, Parveen et al. (1994) defenderam que a biodisponibilidade de Zn diminui com o aumento do pH e com o tempo de aplicação. McBride (1995) ressal tou que a Agência de Proteção Ambiental dos Estados Unidos (USEPA) admite limites mais el evados de metais em solos agrícolas tratados com lodo de esgoto, considerando que a presença de fosfatos, silicatos, óxidos e oxidróxidos presentes no lodo contribuem para a retenção desses metais, evitando contaminação de solos efitotoxidez em cultivos prolongados.

O uso de técnicas de fracionamento permite inferências sobre a origem, forma de ocorrência, biodisponibilidade, fluxos, mobilidade e transporte dos metais, além da fitotoxidez, dinâmica dos elementos-traços e transformações entre as diferentes formas químicas em solos poluídos ou agrícolas (Miller et al., 1986). A biodisponibilidade, a mobilidade e a toxidez de metais, como o Zn, são determinadas pela cinética de distribuição entreas fases dos sol os e seus componentes (Han et al., 2003), situação na qual as extrações seqüenciais podem ajudar na previsão da disponibilidade.

Essa técnica tem-se mostrado bastante útil para avaliação da disponi bilidade e formas de metais em solos tratados com lodo de esgoto (Oliveira et al., 2003; Qiao et al., 2003; Fuentes et al., 2004). Os resultados obtidos são al gumas vezes conflitantes 
em decorrência dos diferentes métodos utilizados, das características diversas dos lodos e da própria complexidade das reações solo-lodo.

Silviera \& Sommers (1977) encontraram que a proporção de Zn disponível extraído por DTPA em sol os incubados com lodo por 28 dias aumentou com o tempo, ocasionando alterações na disponibilidade às plantas. Em concordância com esses dados, Planquart et al. (1999) relataram que o Zn foi o metal de maior disponibilidade em solos aos quais se adicionou composto de lodo de esgoto. No entanto, deve-se salientar, como observado por Barrow (1993), que maior tempo de contato com o solo fará com que o Zn seassocie às formas mais estáveis, por exemplo, óxidos cristalinos. Considerando estas transformações, Parveen et al . (1994) mostram que experimentos que visam estudar as alterações de Zn no solo após aplicação de lodo devem ser realizados por vários meses, uma vez que maior quantidade de Zn deverá ser retida por frações cristalinas e residuais do solo.

Alguns extratores, notadamente a solução do agente quelante DTPA-TEA, também têm sido utilizados para extração dos teores de Zn disponíveis em solos tratados com lodo de esgoto (Hooda \& Alloway, 1994; F uentes et al., 2004; Nascimento et al., 2004). Embora não permita maiores conclusões sobre a mobilidade do $\mathrm{Zn}$ entre frações do solo, o DTPA tem sido bastante utilizado mundialmenteem virtude de sua praticidade e boa correlação com os teores absorvidos por plantas.

Este trabal ho objetivou estudar a redistribuição entrefrações e os teor es disponíveis de Zn extraídos por DTPA em solos incubados com doses de lodo de esgoto.

\section{MATERIAL E MÉTODOS}

F oram col etadas amostras de dois solos do Estado de Pernambuco, nos municípios de Carpina e I pojuca, obtidos do horizonte superficial $(0$ a $20 \mathrm{~cm})$, os quais foram classificados, respectivamente, como Argissolo Amarelo (PA) e Latossolo Amarelo (LA). Estes sol os foram selecionados para o experimento de acordo com seus valores diferentes de argila, matéria orgânica e pH. Antes do início do experimento, as amostras foram caracterizadas química e fisicamente (Quadro 1).

O lodo de esgoto utilizado foi proveniente da Estação de Tratamento de Esgoto Doméstico (ETE) do bairro da Mangueira, Recife, pertencente à COMPESA (Companhia Pernambucana de Saneamento). Após seco, o material foi submetido às digestões: sulfúrica, para determinação de $\mathrm{N}$, e nitricoperclórica, para determinação dos elementos $\mathrm{P}, \mathrm{K}, \mathrm{Ca}, \mathrm{Mg}, \mathrm{Na}, \mathrm{Zn}, \mathrm{Cu}, \mathrm{Mn}, \mathrm{Fe}, \mathrm{Pb}$ e $\mathrm{Cd}$, e combustão em mufla, para determinação do conteúdo de matéria orgânica (Quadro 2).
Quadro 1. Caracterização química e física das amostras de solos utilizadas no experimento

\begin{tabular}{|c|c|c|}
\hline Característica & Argissolo & Latossolo \\
\hline $\mathrm{pH}$ em $\mathrm{H}_{2} \mathrm{O}(1: 2,5)^{(1)}$ & 6,1 & 4,3 \\
\hline C.O. $\left(\mathrm{g} \mathrm{kg}^{-1}\right)^{(2)}$ & 3,92 & 9,63 \\
\hline M.O. $\left(\mathrm{g} \mathrm{kg}^{-1}\right)^{(2)}$ & 6,76 & 16,61 \\
\hline $\mathrm{Ca}\left(\mathrm{cmol}_{\mathrm{c}} \mathrm{dm}^{-3}\right)^{(1)}$ & 2,04 & 1,6 \\
\hline $\mathrm{Mg}\left(\mathrm{cmol}_{\mathrm{c}} \mathrm{dm}^{-3}\right)^{(1)}$ & 2,63 & 0,8 \\
\hline $\mathrm{Al}\left(\mathrm{cmol}_{\mathrm{c}} \mathrm{dm}^{-3}\right)^{(1)}$ & 0,0 & 1,35 \\
\hline $\mathrm{Na}\left(\mathrm{cmol}_{\mathrm{c}} \mathrm{dm}^{-3}\right)^{(1)}$ & 0,15 & 0,14 \\
\hline$P\left(\mathrm{mg} \mathrm{dm}^{-3}\right)^{(1)}$ & 17,6 & 15,1 \\
\hline $\mathrm{K}\left(\mathrm{cmol}_{\mathrm{c}} \mathrm{dm}^{-3}\right)^{(1)}$ & 0,55 & 0,24 \\
\hline $\mathrm{H}+\mathrm{Al}\left(\mathrm{cmol}_{\mathrm{c}} \mathrm{dm}^{-3}\right)^{(1)}$ & 1,90 & 11,22 \\
\hline $\mathrm{Zn}\left(\mathrm{mg} \mathrm{dm}^{-3}\right)^{(3)}$ & 3,5 & 0,9 \\
\hline $\mathrm{Cu}\left(\mathrm{mg} \mathrm{dm}^{-3}\right)^{(3)}$ & 0,6 & 0,6 \\
\hline $\mathrm{Fe}\left(\mathrm{mg} \mathrm{dm}^{-3}\right)^{(3)}$ & 19,1 & 58,6 \\
\hline $\mathrm{Mn}\left(\mathrm{mg} \mathrm{dm}^{-3}\right)^{(3)}$ & 5,2 & 1,8 \\
\hline Zn total $\left(\mathrm{mg} \mathrm{dm}^{-3}\right)^{(4)}$ & 41,7 & 54,8 \\
\hline Cu total $\left(\mathrm{mg} \mathrm{dm}^{-3}\right)^{(4)}$ & 10,5 & 16,7 \\
\hline Fe total $\left(\mathrm{mg} \mathrm{dm}^{-3}\right)^{(4)}$ & $9.090,2$ & $48.765,1$ \\
\hline $\mathrm{Mn}$ total $\left(\mathrm{mg} \mathrm{dm}^{-3}\right)^{(4)}$ & 100,6 & 133,2 \\
\hline Areia $\left(\mathrm{g} \mathrm{kg}^{-1}\right)^{(1)}$ & 760 & 504 \\
\hline Silte $\left(\mathrm{g} \mathrm{kg}^{-1}\right)^{(1)}$ & 69 & 84 \\
\hline Argila $\left(\mathrm{g} \mathrm{kg}^{-1}\right)^{(1)}$ & 171 & 412 \\
\hline
\end{tabular}

(1) Embrapa (1997). (2) Kiehl (1985). (3) Mehlich-1 (De Filippo \& Ribeiro, 1997). ${ }^{(4)}$ United States (1996).

Quadro 2. Caracterização química (teores totais) do lodo de esgoto utilizado no experimento

\begin{tabular}{lr}
\hline Característica & Valor \\
\hline $\mathrm{pH}$ em $\mathrm{H}_{2} \mathrm{O}(1: 2,5)^{(1)}$ & 5,7 \\
$\mathrm{~N}$-total $\left(\mathrm{g} \mathrm{kg}^{-1}\right)^{(2)}$ & 22,5 \\
Carbono $\left(\mathrm{g} \mathrm{kg}^{-1}\right)^{(3)}$ & 285,9 \\
M.O. $\left(\mathrm{g} \mathrm{kg}^{-1}\right)^{(3)}$ & 493,0 \\
Relação C/N-total & 12,7 \\
Cálcio $\left(\mathrm{g} \mathrm{kg}^{-1}\right)^{(2)}$ & 15,3 \\
Magnésio $\left(\mathrm{g} \mathrm{kg}^{-1}\right)^{(2)}$ & 1,9 \\
Sódio $\left(\mathrm{g} \mathrm{kg}^{-1}\right)^{(2)}$ & 1,0 \\
Potássio $\left(\mathrm{g} \mathrm{kg}^{-1}\right)^{(2)}$ & 1,9 \\
Fósforo $\left(\mathrm{g} \mathrm{kg}^{-1}\right)^{(2)}$ & 4,7 \\
Manganês $\left(\mathrm{mg} \mathrm{kg}^{-1}\right)^{(2)}$ & 127 \\
Zinco $\left(\mathrm{mg} \mathrm{kg}^{-1}\right)^{(2)}$ & 865 \\
Chumbo $\left(\mathrm{mg} \mathrm{kg}^{-1}\right)^{(2)}$ & 350 \\
Cobre $\left(\mathrm{mg} \mathrm{kg}^{-1}\right)^{(2)}$ & 128 \\
Cádmio $\left(\mathrm{mg} \mathrm{kg}^{-1}\right)^{(2)}$ & 31 \\
Ferro $\left(\mathrm{mg} \mathrm{kg}^{-1}\right)^{(2)}$ & 15.816
\end{tabular}

(1) Embrapa (1997). ${ }^{(2)}$ Embrapa (1999). ${ }^{(3)}$ Kiehl (1985).

As amostras de solo e do lodo de esgoto, isoladamente, foram secas, homogeneizadas e passadas em peneira de $2 \mathrm{~mm}$ de abertura de malha. As doses de lodo utilizadas foram estabel ecidas em 
propor ções de 0,$0 ; 0,25 ; 0,50 ; 1,00$ e 1,50 vez olimite máximo aceitável da concentração de Zn após a aplicação de lodo de esgoto ao solo, que corresponde a $140 \mathrm{~kg} \mathrm{ha}^{-1} \mathrm{ano}^{-1}$, segundo a United States Environmental Protection Agency - USE PA (United States, 1993). As doses utilizadas, considerando a concentração de Zn nolodo utilizado no experimento, foram equivalentes, respectivamente, a 0; 40,5; 81; 162 e $243 \mathrm{Mg} \mathrm{ha}^{-1}$.

As amostras de sol o mais lodo, com volume final de $3,5 \mathrm{dm}^{3}$, foram incubadas em laboratório, em sacos plásticos com umidade mantida próxima à capacidade de campo. Nos interval os de 30, 60, 90, 120,150 e 180 dias, foram coletadas subamostras para determinação dos teores disponíveis de Zn extraídos com DTPA e os teores deste el emento em frações do solo. As subamostras coletadas foram submetidas à secagem em estufa a $65^{\circ} \mathrm{C}$, maceradas e passadas em peneira com $0,5 \mathrm{~mm}$ de malha.

O Zn disponível foi extraído pelo DTPA (Lindsay \& N orvell, 1978), enquanto a extração seqüencial foi baseada no método de Shuman (1985), com exceção da fração óxido de F e amorfo, que foi obtida pelo método de Chao \& Zhou (1983). Esse fracionamento separa Zn nas frações trocáveis, ligadas à matéria orgânica, óxido de Fe amorfo e óxido de Fe cristalino. Os teores totais de Zn foram obtidos por digestão ácida em forno microondas, de acordo com U nited States (1996).
O ensaio foi montado em delineamento intei ramente casual izado, utilizando-se dois sol os e cinco doses de lodo, com três repetições, sendo os dados submetidos à análise de variância, de regressão, correlação e teste de Tukey, utilizandose o software SAEG (Sistema de Análise Estatística e Genética) da Universidade F ederal de Viçosa.

\section{RESULTADOS E DISCUSSÃO}

Os teores de Zn total e disponível extraído por DTPA, para todos os períodos de incubação, aumentaram com a aplicação das doses de lodo de esgoto (Quadro 3).

Em ambos os solos, os aumentos nos teores de Zn disponível foram lineares em relação às doses aplicadas (Figura 1), embora os teores extraídos pelo DTPA tenham sido maiores no Argissolo (Quadro 3). Este resultado indica a forte dependência do Zn à adsorção pela fração mineral do solo (Machado \& Pavan, 1987; Nascimento \& Fontes, 2004), mais pronunciada no L atossolo, provavel mente em razão dos maiores teores de argila e maior presença de óxidos.

Quanto aos teores totais, mesmo a mais al ta dose de lodo utilizada neste trabalho (243 $\mathrm{Mg} \mathrm{ha}^{-1}$ ), equivalente a 1,5 vez a máxima dose recomendada

Quadro 3. Teores de Zn disponível extraídos por DTPA e teor total após aplicação de doses de lodo de esgoto em diferentes dias de incubação e percentagem relativa em relação ao $\mathrm{Zn}$ total nos solos

\begin{tabular}{|c|c|c|c|c|c|c|c|c|c|c|c|c|c|}
\hline \multirow{3}{*}{$\begin{array}{c}\text { Dose } \\
\mathrm{Mg} \mathrm{ha}^{-1}\end{array}$} & \multicolumn{12}{|c|}{ Dia de incubação } & \multirow{3}{*}{$\frac{\text { Total }}{\text { mg dm-3 }}$} \\
\hline & \multicolumn{2}{|c|}{30} & \multicolumn{2}{|c|}{60} & \multicolumn{2}{|c|}{90} & \multicolumn{2}{|c|}{120} & \multicolumn{2}{|c|}{150} & \multicolumn{2}{|c|}{180} & \\
\hline & $\mathrm{mg} \mathrm{dm}^{-3}$ & $\%$ & $\mathrm{mg} \mathrm{dm}-3$ & $\%$ & $\mathrm{mg} \mathrm{dm}^{-3}$ & $\%$ & $\mathrm{mg} \mathrm{dm}^{-3}$ & $\%$ & $\mathrm{mg} \mathrm{dm}^{-3}$ & $\%$ & $\mathrm{mg} \mathrm{dm}^{-3}$ & $\%$ & \\
\hline \multicolumn{14}{|c|}{ Argissolo } \\
\hline 0 & 4,8 & 8,7 & 3,3 & 6,1 & 3,6 & 6,5 & 2,8 & 5,2 & 3,0 & 5,5 & 3,2 & 5,9 & 41,7 \\
\hline 40,5 & 20,3 & 27,4 & 17,3 & 23,4 & 17,8 & 24,0 & 14,6 & 19,7 & 14,3 & 19,2 & 15,7 & 21,2 & 57,4 \\
\hline 81 & 33,8 & 38,0 & 28,3 & 31,9 & 32,8 & 36,9 & 23,5 & 26,5 & 22,6 & 25,4 & 30,1 & 33,9 & 76,2 \\
\hline 162 & 51,7 & 42,6 & 50,6 & 41,7 & 58,6 & 48,3 & 45,1 & 37,2 & 41,8 & 34,4 & 49,2 & 40,6 & 108,4 \\
\hline 243 & 63,9 & 42,0 & 87,7 & 57,6 & 77,3 & 50,7 & 59,1 & 38,8 & 55,8 & 36,6 & 58,6 & 38,5 & 148,1 \\
\hline M édia & 34,9 & 40,1 & 37,5 & 37,4 & 38,0 & 40 & 29,0 & 34,5 & 27,5 & 33,7 & 31,4 & 37,1 & 86,4 \\
\hline C.V. (\%) & 2,55 & & 2,17 & & 2,81 & & 3,31 & & 3,96 & & 4,41 & & 3,4 \\
\hline \multicolumn{14}{|c|}{ Latossolo } \\
\hline 0 & 1,1 & 1,9 & 0,8 & 1,5 & 0,7 & 1,3 & 0,4 & 0,72 & 0,5 & 0,9 & 0,7 & 1,3 & 54,7 \\
\hline 40,5 & 11,2 & 15,1 & 10,3 & 13,9 & 9,4 & 12,7 & 7,7 & 10,4 & 7,6 & 10,3 & 7,2 & 9,7 & 74,1 \\
\hline 81 & 13,6 & 15,3 & 18,4 & 20,7 & 17,8 & 20,0 & 15,6 & 17,5 & 14,4 & 16,2 & 13,5 & 15,2 & 88,8 \\
\hline 162 & 26 & 21,5 & 34,9 & 28,8 & 42,1 & 34,7 & 26,6 & 21,9 & 27,6 & 22,7 & 25,1 & 20,7 & 121,3 \\
\hline 243 & 35,7 & 23,4 & 57,7 & 37,9 & 53,0 & 34,8 & 47,8 & 31,4 & 41,9 & 27,5 & 38,8 & 25,5 & 152,3 \\
\hline Média & 17,5 & 27,5 & 24,4 & 29,8 & 24,6 & 30,6 & 19,6 & 26,9 & 18,41 & 26,8 & 17,1 & 26,2 & 98,3 \\
\hline C.V. (\%) & 4,73 & & 3,76 & & 3,72 & & 6,06 & & 5,99 & & 5,14 & & 6,4 \\
\hline
\end{tabular}




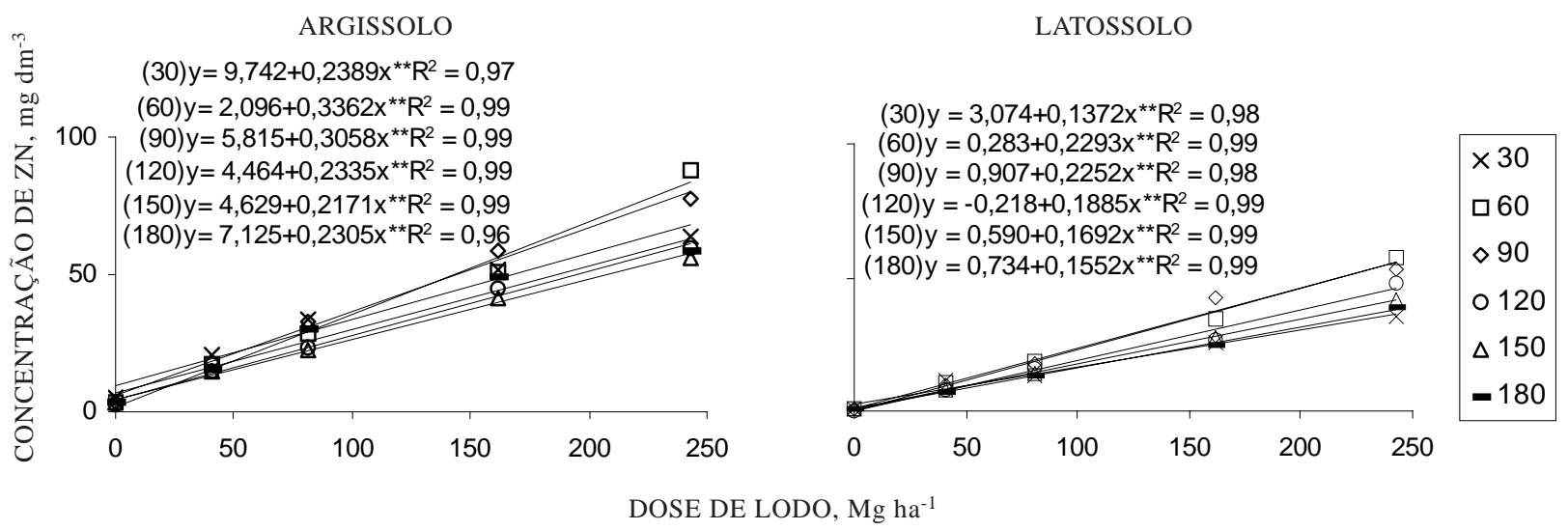

Figura 1. E feito de doses de lodo de esgoto sobre os teores deZn extraído por DTPA em diferentes períodos de incubação (30, 60, 90, 120, 150 e 180 dias).

pela USEPA (United States, 1993), representou teores de $\mathrm{Zn}$ nos solos abaixo da concentração máxima permitida do elemento pela legislação americana (1.500 $\left.\mathrm{mg} \mathrm{kg}^{-1}\right)$, bem como pela Comunidade Européia (300 $\mathrm{mg} \mathrm{kg}^{-1}$ ) (KabataPendias \& Adriano, 1995) e pelo valor de alerta (menor concentração de $\mathrm{Zn}$ em solos que causa alguma fitotoxidez $=300 \mathrm{mg} \mathrm{kg}^{-1}$ ), sugerido pela Companhia de Tecnol ogia de Saneamento Ambiental do Estado de São Paulo (CETESB, 2001).

As doses de lodo da COMPESA que proporcionam máxima eficiência agronômica para a cultura do milho, $26 \mathrm{Mg} \mathrm{ha}^{-1}$ (Gomes, 2004), foram bem inferiores às testadas no presente trabalho e poderiam ser aplicadas anualmente sem maiores riscos quanto ao aumento da disponibilidade de Zn. No entanto, deve-sealertar que os dados do presente trabal ho foram obtidos em condições controladas e a dinâmica de Zn após aplicações seguidas de lodo utilizado necessita também ser validada para condições de campo.

Provavel mente, em decorrência da mineralização da matéria orgânica, foram observados aumentos nos teores médios deZn disponível nas determinações efetuadas aos 60 e 90 dias (Quadro 3); no entanto, apesar dessas flutuações com os meses de incubação, não foi observado efeito significativo $(p<0,05)$ sobre a disponibilidade do el emento. Corroborando esta afirmação, Araújo (2004) encontrou aumento de produção de matéria seca e ausência de fitotoxidez de $\mathrm{Zn}$ em plantas de milho cultivadas em solos incubados por 180 dias com a mais alta dose de lodo utilizada neste experimento (243 $\mathrm{Mg} \mathrm{ha}^{-1}$ ). Resultado semel hante foi encontrado por McGrath et al. (2000), em sol os que receberam doses de lodo por 23 anos, e por Nyamangara \& Mzezewa (1999), em solos que receberem lodo por 19 anos. Contrariamente a esses resultados, Silviera \& Sommers (1977) e Oliveira et al. (2003) observaram aumentona disponibilidade de Zn em sol os incubados por 28 dias e 160 semanas, respectivamente.
Portanto, os resultados encontrados neste e em outros trabalhos na literatura não apresentaram dara tendência quantoa mudanças na disponibilidade de $\mathrm{Zn}$ com tempos de contato solo-lodo, com variações, inclusive, quando a extração foi efetuada em diferentes temperaturas do solo (Hooda \& Alloway, 1994).

Houve diminuição significativa do $\mathrm{pH}$ das amostras de solo que receberam adição de lodo de esgoto de acordo com o tempo de incubação (Quadro 4). Esta redução está relacionada, provavelmente, aos processos de decomposição da matéria orgânica adicionada, sendo mais expressiva para as maiores doses de lodo no Argissolo, que apresentava $\mathrm{pH}$ inicial mais el evado que o Latossolo (Quadro 1).

Anal isando as transformações nas frações de Zn durante os 180 dias de incubação, observaram-se respostas diferenciadas entre os dois solos para os teores trocáveis do el emento. Enquanto no Argissolo (Quadro 5) houve aumento significativo no $\mathrm{Zn}$ trocável a partir da dose $81 \mathrm{Mg} \mathrm{ha}^{-1}$ com o decorrer do período de incubação, os teores trocáveis do elemento no Latossolo (Quadro 6) apresentaram diferença entre 30 e 180 dias de contato apenas para a dose mais alta de lodo. Este resultado corrobora a menor extração de Zn pelo DTPA no Latossolo, comentada anteriormente, e as diferenças na capacidade-tampão dos solos, com conseqüente maior disponibilização do $\mathrm{Zn}$ adicionado via lodo para o Argissolo.

A diferença de $\mathrm{pH}$ entre os sol os (Quadro 1), bem como a própria diminuição de $\mathrm{pH}$ relacionada com o aumento do tempo de contato solo-lodo, é outro importante fator que influencia os teores trocáveis de Zn. O decréscimo de uma unidade depH aumenta a solubilidade dos metais em dez vezes, aumentando a solubilidade e potencial delixiviação desses metais do perfil de solo, especial mente para metais de maior mobilidade como o Zn (Planquart et al., 1999). 


\section{Quadro 4. Valores de pH e equações de regressão entre pH e dias de incubação para dois solos incubados com lodo de esgoto}

\begin{tabular}{|c|c|c|c|c|c|c|c|c|}
\hline \multirow{2}{*}{ Dose } & \multicolumn{6}{|c|}{ Dia de incubação } & \multirow{2}{*}{ Regressão } & \multirow{2}{*}{$\mathbf{R}^{2}$} \\
\hline & 30 & 60 & 90 & 120 & 150 & 180 & & \\
\hline $\mathrm{Mg} \mathrm{ha}^{-1}$ & \multicolumn{8}{|c|}{ Argissolo } \\
\hline 0 & 6,0 & 6,4 & 6,5 & 6,4 & 6,3 & 6,0 & $\hat{y}=6,712+0,577^{* *} x-0,0031^{* *} x^{2}$ & 0,99 \\
\hline 40,5 & 4,8 & 4,8 & 4,9 & 4,9 & 4,8 & 4,7 & $\hat{y}=4,214+0,151^{*} x-0,0082^{* *} x^{2}$ & 0,84 \\
\hline 81 & 5,0 & 4,8 & 4,7 & 4,7 & 4,7 & 4,6 & $\hat{y}=5,033-0,0026^{* *} x$ & 0,87 \\
\hline 162 & 4,9 & 4,9 & 4,7 & 4,8 & 4,3 & 4,1 & $\hat{y}=5,193-0,0054^{* * x}$ & 0,85 \\
\hline 243 & 5,4 & 4,9 & 4,8 & 4,7 & 4,4 & 4,3 & $\hat{y}=5,433-0,0066^{* *} x$ & 0,90 \\
\hline \multicolumn{9}{|c|}{ Latossolo } \\
\hline 0 & 4,6 & 4,2 & 4,1 & 4,5 & 4,1 & 4,0 & $\hat{y}=\bar{y}=28,68$ & ns \\
\hline 40,5 & 4,8 & 4,5 & 4,5 & 4,4 & 4,2 & 4,1 & $\hat{y}=4,862-0,0042^{* *} x$ & 0,91 \\
\hline 81 & 4,9 & 4,6 & 4,5 & 4,6 & 4,2 & 4,3 & $\hat{y}=4,915-0,0039^{* *} x$ & 0,84 \\
\hline 162 & 5,1 & 5,1 & 4,8 & 4,9 & 4,6 & 4,6 & $\hat{y}=5,249-0,0036^{* *} x$ & 0,84 \\
\hline 243 & 5,3 & 5,1 & 4,8 & 4,8 & 4,5 & 4,4 & $\hat{y}=5,436-0,0059^{* *} x$ & 0,95 \\
\hline
\end{tabular}

*,** e ns: Significativos a 5 e 1 \%, respectivamente e não-significativo.

Os aumentos nos teores de Zn trocável parecem estar relacionados com a mineralização da matéria orgânica do lodo, com subseqüente adsorção de parte do metal pela matriz mineral do solo, como indicado pela correlação negativa entre as frações (trocável e matéria orgânica), quevariaram entre-0,82* e-0,93**, para oLatossolo, eentre-0,86* e-0,98**, para oArgissolo.

Os teores de Zn ligado à fração matéria orgânica foram significativamenter reduzi dos após 180 dias de incubação, para ambos os solos, em todas as doses de lodo de esgoto utilizadas (Quadros 5 e 6). Esta diminuição está relacionada com a estabilidade dos metais em solos tratados com lodo de esgoto, notadamente em relação à taxa e à quantidade de decomposição do lodo, com conseqüente fornecimento de metais originalmente complexados pelos compostos orgânicos (Silviera \& Sommers, 1977). Parveen et al. (1994) encontraram aumento dos teores de $\mathrm{Zn}$ organicamente ligado com o aumento do tempo de incubação, em solos de pH neutro; em concordância com os resultados do presentetrabalho, esses autores não observaram este efeito em solos ácidos.

É provável que o lodo utilizado apresente baixa relação ácido húmico/ácido fúlvico, tal como observada no lodo de esgoto utilizado por Oliveira et al. (2003). Neste caso, o Zn nas amostras estaria complexado, principalmente, por agentes quelantes de baixo peso molecular. Como demonstrado por Stevenson \& Fitch (1989), a estabilidade dos complexos orgânicos com o Zn diminui, consideravelmente, com o aumento da acidez do sol o provocada pela adição do lodo (Quadro 4), resultando em transferência do elemento da fração orgânica para outros compartimentos do solo. Foram observadas boas correlações entre a diminuição do Zn ligado organicamenteea transferência do el emento para frações residuais em ambos os solos: $-0,93^{* *}$ e $-0,98 * *$, para o Latossol oeA rgissolo, respectivamente.

Os resultados indicam que a maior parte do $\mathrm{Zn}$ orgânico adicionado via lodo de esgoto foi transferida para formas de baixa disponibilidade, como é o caso da fração residual. Estas frações (orgânica eresi dual) foram os sítios preferenciais de concentração de Zn em ambos os sol os (Quadros 5 e 6).

A fração óxido de $\mathrm{Fe}$ amorfo apresentou os menores percentuais de retenção de Zn para os dois solos (Quadros 5 e 6). A pesar da diminuição dos teores de Zn nesta fração, com o aumento das doses aplicadas, os teores médios do elemento nos diferentes tempos de incubação apresentaram clara tendência de aumento. Comportamento idêntico foi observado para a fração óxido de Fe cristalino. É possível que, em solos de $\mathrm{pH}$ mais elevado que os utilizados nestetrabalho, a transferência de Zn para essas frações seja ainda maior, com conseqüente diminuição dos teores disponíveis (Parveen et al., 1994). Nascimento et al. (2002) observaram aumento de 4 a 6 vezes no $Z n$ ligado à fração óxido de $\mathrm{Fe}$ amorfo, em solos que receberam calagem, relativamente a sol os de reação ácida. Neste aspecto, a apl icação de cal cário em sol os que receberam altas quantidades de lodo de esgoto deveser considerada, visando à diminuição de possíveis teores fitotóxicos. 
Quadro 5. E feito do tempo de incubação na redistribuição de Zn entre frações no Argissolo tratado com doses de lodo de esgoto e percentagem relativa ao $\mathrm{Zn}$ total no solo

\begin{tabular}{|c|c|c|c|c|c|c|c|c|c|c|c|c|}
\hline \multirow{3}{*}{$\begin{array}{c}\text { Dose } \\
\text { Mg ha-1 }\end{array}$} & \multirow{3}{*}{$\begin{array}{c}\text { Tempo } \\
\text { dia }\end{array}$} & \multicolumn{11}{|c|}{ Fração } \\
\hline & & \multicolumn{2}{|l|}{$\mathbf{T r}$} & \multicolumn{2}{|c|}{ MO } & \multicolumn{2}{|c|}{ OxF eA } & \multicolumn{2}{|c|}{ OxFeC } & \multicolumn{2}{|l|}{$\mathbf{R}$} & \multirow{2}{*}{$\begin{array}{c}\text { Total } \\
\mathrm{mg} \mathrm{dm}^{-3}\end{array}$} \\
\hline & & $\mathrm{mg} \mathrm{dm}^{-3}$ & $\%$ & $\mathrm{mg} \mathrm{dm}^{-3}$ & $\%$ & $\mathrm{mg} \mathrm{dm}^{-3}$ & $\%$ & $\mathrm{mg} \mathrm{dm}^{-3}$ & $\%$ & $\mathrm{mg} \mathrm{dm}-3$ & $\%$ & \\
\hline \multirow[t]{6}{*}{0} & 30 & nd & 0,0 & $8,4 \mathrm{~b}$ & 20,1 & $0,7 \mathrm{a}$ & 1,7 & $2,5 \mathrm{a}$ & 5,9 & $30,1 \mathrm{a}$ & 72,3 & $41,7 a$ \\
\hline & 60 & nd & 0,0 & $10,3 \mathrm{a}$ & 24,6 & nd & 0,0 & $1,9 a b$ & 4,6 & 29,5 a & 70,7 & $41,7 \mathrm{a}$ \\
\hline & 90 & nd & 0,0 & 8,7 b & 20,8 & $0,4 \mathrm{~b}$ & 1,0 & $2,4 a$ & 5,7 & 30,2 a & 72,4 & $41,7 \mathrm{a}$ \\
\hline & 120 & nd & 0,0 & $10,1 \mathrm{a}$ & 24,2 & $0,1 \mathrm{c}$ & 0,2 & 1,2 bc & 2,8 & $30,3 \mathrm{a}$ & 72,8 & $41,7 \mathrm{a}$ \\
\hline & 150 & nd & 0,0 & $8,9 \mathrm{~b}$ & 21,4 & nd & 0,0 & $0,9 \mathrm{c}$ & 2,1 & 31,8 a & 76,4 & $41,7 \mathrm{a}$ \\
\hline & 180 & nd & 0,0 & $5,6 \mathrm{c}$ & 13,4 & nd & 0,0 & $2,2 \mathrm{a}$ & 5,4 & $33,9 a$ & 81,3 & $41,7 \mathrm{a}$ \\
\hline Média & & - & 0,0 & 8,6 & 20,8 & 0,2 & 0,5 & 1,8 & 4,4 & 31,0 & 74,3 & 41,7 \\
\hline C.V. (\%) & & - & & 3,7 & & 20,8 & & 17,6 & & 6,4 & & 4,6 \\
\hline \multirow[t]{6}{*}{40,5} & 30 & nd & 0,0 & $31,3 \mathrm{~b}$ & 54,5 & $1,1 \mathrm{a}$ & 2,0 & $3,1 \mathrm{a}$ & 5,4 & $21,9 \mathrm{c}$ & 38,1 & 57,4 a \\
\hline & 60 & $2,1 \mathrm{ab}$ & 3,8 & $34,2 \mathrm{a}$ & 59,5 & nd & 0,0 & $2,3 a$ & 4,0 & $18,7 \mathrm{c}$ & 32,7 & $57,4 a$ \\
\hline & 90 & $3,3 \mathrm{a}$ & 5,7 & $24,5 d$ & 42,6 & $0,3 \mathrm{~b}$ & 0,5 & $2,9 a$ & 5,2 & 26,4 bc & 45,9 & $57,4 a$ \\
\hline & 120 & nd & 0,0 & $27,1 \mathrm{c}$ & 47,1 & $0,2 \mathrm{c}$ & 0,3 & $1,4 \mathrm{~b}$ & 2,5 & $28,7 \mathrm{abc}$ & 50,0 & $57,4 a$ \\
\hline & 150 & nd & 0,0 & $19,8 \mathrm{e}$ & 34,4 & nd & 0,0 & $1,4 \mathrm{~b}$ & 2,4 & $36,2 a b$ & 63,8 & $57,4 a$ \\
\hline & 180 & $1,8 \mathrm{~b}$ & 3,1 & $12,3 \mathrm{f}$ & 21,4 & nd & 0,0 & $2,7 \mathrm{a}$ & 4,7 & 40,6 a & 70,8 & $57,4 a$ \\
\hline Média & & 1,2 & 2,1 & 24,8 & 43,3 & 0,3 & 0,5 & 2,3 & 4,0 & 28,8 & 50,1 & 57,4 \\
\hline C.V. & & 35,7 & & 3,6 & & 6,9 & & 13,3 & & 15,4 & & 8,0 \\
\hline \multirow[t]{6}{*}{81} & 30 & $1,9 \mathrm{~d}$ & 2,5 & $48,3 \mathrm{~b}$ & 63,4 & $2,2 \mathrm{a}$ & 2,9 & $4,6 a$ & 6,0 & 19,2 b & 25,2 & $76,2 \mathrm{a}$ \\
\hline & 60 & $5,3 \mathrm{~b}$ & 7,0 & $53,6 a$ & 70,3 & nd & 0,0 & $3,0 \mathrm{~b}$ & 3,9 & $14,3 \mathrm{~b}$ & 18,7 & $76,2 \mathrm{a}$ \\
\hline & 90 & $8,9 a$ & 11,7 & $37,7 \mathrm{c}$ & 49,5 & $0,9 \mathrm{~b}$ & 1,2 & $4,2 \mathrm{a}$ & 5,5 & $24,5 \mathrm{~b}$ & 32,1 & 76,2 a \\
\hline & 120 & 4,3 bc & 5,6 & $41,6 \mathrm{c}$ & 54,6 & $0,3 \mathrm{c}$ & 0,4 & $1,7 \mathrm{c}$ & 2,2 & $28,3 \mathrm{~b}$ & 37,2 & 76,2 a \\
\hline & 150 & $2,4 \mathrm{~cd}$ & 3,2 & $23,2 d$ & 30,4 & nd & 0,0 & $1,2 \mathrm{~d}$ & 1,6 & $49,4 a$ & 64,9 & $76,2 \mathrm{a}$ \\
\hline & 180 & $5,9 \mathrm{~b}$ & 7,7 & 16,9 e & 22,2 & nd & 0,0 & $2,9 \mathrm{~b}$ & 3,8 & $50,5 a$ & 66,3 & 76,2 a \\
\hline Média & & 4,8 & 6,3 & 36,8 & 48,4 & 0,6 & 0,8 & 2,9 & 3,8 & 31,0 & 40,7 & 76,2 \\
\hline C.V. (\%) & & 14,8 & & 4,8 & & 23,1 & & 5,8 & & 19,2 & & 6,6 \\
\hline \multirow[t]{6}{*}{162} & 30 & $4,0 \mathrm{~d}$ & 3,7 & $77,3 \mathrm{a}$ & 71,3 & $4,6 a$ & 4,3 & $6,9 a$ & 6,3 & $15,6 \mathrm{c}$ & 14,4 & $108,4 \mathrm{a}$ \\
\hline & 60 & $9,6 \mathrm{c}$ & 8,9 & $82,3 a$ & 75,9 & $0,0 \mathrm{c}$ & 0,0 & $3,4 \mathrm{c}$ & 3,1 & $13,0 \mathrm{c}$ & 12,0 & $108,4 \mathrm{a}$ \\
\hline & 90 & $15,8 \mathrm{~b}$ & 14,7 & $56,4 \mathrm{~b}$ & 52,0 & $1,6 \mathrm{~b}$ & 1,5 & $5,4 \mathrm{~b}$ & 5,0 & $29,0 \mathrm{~b}$ & 26,7 & $108,4 \mathrm{a}$ \\
\hline & 120 & $11,9 \mathrm{c}$ & 11,0 & $63,8 \mathrm{~b}$ & 58,8 & $0,3 \mathrm{c}$ & 0,3 & $2,4 \mathrm{~d}$ & 2,3 & $29,9 \mathrm{~b}$ & 27,6 & $108,4 \mathrm{a}$ \\
\hline & 150 & $9,6 \mathrm{c}$ & 8,9 & $35,9 \mathrm{c}$ & 33,2 & nd & 0,0 & $1,7 \mathrm{e}$ & 1,6 & $61,0 a$ & 56,3 & $108,4 \mathrm{a}$ \\
\hline & 180 & $19,6 a$ & 18,0 & $26,6 d$ & 24,5 & nd & 0,0 & $3,7 \mathrm{c}$ & 3,4 & $58,6 a$ & 54,1 & $108,4 a$ \\
\hline Média & & 11,8 & 10,9 & 57,8 & 52,6 & 1,1 & 1,0 & 3,9 & 3,6 & 34,5 & 31,8 & 108,4 \\
\hline C.V. (\%) & & 7,3 & & 4,9 & & 17,9 & & 4,2 & & 11,1 & & 2,0 \\
\hline \multirow[t]{6}{*}{243} & 30 & $4,9 \mathrm{c}$ & 3,3 & 104,2 b & 70,3 & $8,0 a$ & 5,4 & $10,1 \mathrm{a}$ & 6,9 & $20,8 \mathrm{~cd}$ & 14,0 & $148,1 \mathrm{a}$ \\
\hline & 60 & $17,3 \mathrm{~b}$ & 11,7 & $114,5 \mathrm{a}$ & 77,3 & $0,2 \mathrm{c}$ & 0,1 & $4,2 \mathrm{c}$ & 2,8 & $11,9 \mathrm{~d}$ & 8,1 & $148,1 \mathrm{a}$ \\
\hline & 90 & $27,3 a$ & 18,5 & $83,6 c$ & 56,4 & $2,8 \mathrm{~b}$ & 1,9 & $8,4 d$ & 5,7 & $26,0 \mathrm{c}$ & 17,5 & $148,1 \mathrm{a}$ \\
\hline & 120 & $18,2 \mathrm{a}$ & 12,3 & $76,9 \mathrm{~d}$ & 51,9 & $0,6 \mathrm{c}$ & 0,4 & $3,2 \mathrm{~d}$ & 2,1 & $49,3 \mathrm{~b}$ & 33,2 & $148,1 \mathrm{a}$ \\
\hline & 150 & $12,0 \mathrm{bc}$ & 8,1 & $52,0 \mathrm{e}$ & 35,1 & nd & 0,0 & $2,2 \mathrm{e}$ & 1,5 & 81,8 a & 55,2 & $148,1 \mathrm{a}$ \\
\hline & 180 & $27,1 \mathrm{a}$ & 18,3 & $43,1 \mathrm{f}$ & 29,1 & nd & 0,0 & $4,9 \mathrm{c}$ & 3,4 & $72,9 \mathrm{a}$ & 49,2 & 148,1 a \\
\hline Média & & 17,8 & 12,0 & 79,06 & 53,4 & 1,9 & 1,3 & 5,5 & 3,7 & 43,7 & 29,5 & 148,1 \\
\hline C.V. (\%) & & 17,5 & & 2,87 & & 14,0 & & 5,2 & & 10,3 & & 2,6 \\
\hline
\end{tabular}

nd = não detectado. Médias seguidas pela mesma letra, nas colunas, dentro de cada dose, não diferem entre si pelo teste Tukey a 1 \%. Tr: Trocável; MO: Ligada a matéria orgânica; OxFeA: Óxido de Ferro Amorfo; OxFeC: Óxido de Ferro Cristalino; R: Residual. 
Quadro 6. Efeito do tempo de incubação na redistribuição de Zn entre frações no Latossolo tratado com doses de lodo de esgoto

\begin{tabular}{|c|c|c|c|c|c|c|c|c|c|c|c|c|}
\hline \multirow{3}{*}{$\begin{array}{c}\text { Dose } \\
\text { Mg ha-1 }^{\text {Dg }}\end{array}$} & \multirow{3}{*}{$\begin{array}{c}\text { Tempo } \\
\text { dia }\end{array}$} & \multicolumn{11}{|c|}{ Fração } \\
\hline & & \multicolumn{2}{|c|}{$\mathbf{T r}$} & \multicolumn{2}{|c|}{ MO } & \multicolumn{2}{|c|}{ OxFeA } & \multicolumn{2}{|c|}{ OxFeC } & \multicolumn{2}{|l|}{$\mathbf{R}$} & \multirow{2}{*}{$\begin{array}{c}\text { Total } \\
\mathrm{mg} \mathrm{dm}^{-3}\end{array}$} \\
\hline & & $\mathrm{mg} \mathrm{dm}^{-3}$ & $\%$ & $\mathrm{mg} \mathrm{dm}^{-3}$ & $\%$ & $\mathrm{mg} \mathrm{dm}^{-3}$ & $\%$ & $\mathrm{mg} \mathrm{dm}^{-3}$ & $\%$ & $\mathrm{mg} \mathrm{dm}-3$ & $\%$ & \\
\hline \multirow[t]{6}{*}{0} & 30 & nd & 0,0 & $6,0 \mathrm{~b}$ & 10,9 & $0,2 d$ & 0,3 & $2,1 \mathrm{~b}$ & 3,8 & 46,5 a & 85,0 & $54,7 \mathrm{a}$ \\
\hline & 60 & nd & 0,0 & $6,8 a$ & 12,5 & nd & 0,0 & $2,1 \mathrm{~b}$ & 3,9 & 45,8 a & 83,7 & $54,7 \mathrm{a}$ \\
\hline & 90 & nd & 0,0 & $5,9 \mathrm{~b}$ & 10,8 & $0,3 a$ & 0,5 & $2,7 \mathrm{a}$ & 4,9 & 45,9 a & 83,8 & 54,7 a \\
\hline & 120 & nd & 0,0 & $6,8 \mathrm{a}$ & 12,4 & $0,0 \mathrm{c}$ & 0,0 & $1,1 \mathrm{C}$ & 2,0 & 46,8 a & 85,6 & 54,7 a \\
\hline & 150 & nd & 0,0 & $5,5 \mathrm{~b}$ & 10,0 & $0,0 \mathrm{c}$ & 0,0 & $0,8 \mathrm{c}$ & 1,4 & 48,5 a & 88,5 & $54,7 \mathrm{a}$ \\
\hline & 180 & nd & 0,0 & $3,1 \mathrm{c}$ & 5,6 & $0,0 \mathrm{c}$ & 0,0 & $2,4 a b$ & 4,4 & 49,2 a & 89,9 & 54,7 a \\
\hline Média & & - & 0 & 5,67 & 10,3 & 0,08 & 0,14 & 1,9 & 3,40 & 47,1 & 86,1 & 54,75 \\
\hline C.V. (\%) & & - & & 4,79 & & 41,15 & & 10,8 & & 5,1 & & 4,7 \\
\hline \multirow[t]{6}{*}{40,5} & 30 & $5,7 \mathrm{c}$ & 7,7 & $13,4 \mathrm{~d}$ & 18,1 & $2,0 \mathrm{a}$ & 2,7 & $3,5 a b$ & 4,8 & $49,5 \mathrm{a}$ & 66,8 & $74,1 \mathrm{a}$ \\
\hline & 60 & $6,6 \mathrm{~b}$ & 8,9 & $20,7 \mathrm{~b}$ & 27,9 & $0,1 \mathrm{c}$ & 0,1 & $2,7 \mathrm{~d}$ & 3,7 & 43,9 a & 59,3 & $74,1 \mathrm{a}$ \\
\hline & 90 & $7,7 \mathrm{a}$ & 10,4 & $16,1 \mathrm{c}$ & 21,7 & $0,6 \mathrm{~b}$ & 0,9 & $3,6 a$ & 4,8 & $46,1 \mathrm{a}$ & 62,2 & $74,1 \mathrm{a}$ \\
\hline & 120 & $4,7 d$ & 6,4 & 24,1 a & 32,6 & $0,1 \mathrm{c}$ & 0,1 & $1,5 \mathrm{c}$ & 2,1 & $43,6 \mathrm{a}$ & 58,9 & 74,1 a \\
\hline & 150 & $3,9 \mathrm{e}$ & 5,2 & $13,6 \mathrm{~d}$ & 18,4 & $0,0 \mathrm{c}$ & 0,0 & $1,2 \mathrm{C}$ & 1,6 & 55,4 a & 74,8 & 74,1 a \\
\hline & 180 & $5,8 \mathrm{c}$ & 7,9 & $10,4 \mathrm{e}$ & 14,1 & $0,0 \mathrm{c}$ & 0,0 & $2,8 \mathrm{ab}$ & 3,7 & $55,1 \mathrm{a}$ & 74,3 & $74,1 \mathrm{a}$ \\
\hline Média & & 5,7 & 7,7 & 16,4 & 22,1 & 0,5 & 0,6 & 2,6 & 3,4 & 49,0 & 66,0 & 74,1 \\
\hline C.V. (\%) & & 3,5 & & 3,9 & & 11,5 & & 11,9 & & 13,5 & & 8,6 \\
\hline \multirow[t]{6}{*}{81} & 30 & $11,0 \mathrm{~b}$ & 12,4 & $29,7 \mathrm{c}$ & 33,5 & $3,7 \mathrm{a}$ & 4,2 & $5,7 a$ & 6,5 & $38,6 \mathrm{bc}$ & 43,5 & 88,8 a \\
\hline & 60 & $11,0 \mathrm{~b}$ & 12,4 & 49,0 a & 55,2 & $0,5 \mathrm{c}$ & 0,6 & $2,8 \mathrm{~b}$ & 3,2 & $25,4 \mathrm{c}$ & 28,6 & $88,8 a$ \\
\hline & 90 & $14,2 \mathrm{a}$ & 16,0 & $30,3 \mathrm{c}$ & 34,1 & $2,0 \mathrm{~b}$ & 2,3 & $5,7 a$ & 6,5 & 36,6 bc & 41,2 & 88,8 a \\
\hline & 120 & $9,9 \mathrm{bc}$ & 11,2 & $36,3 d$ & 40,8 & $0,4 \mathrm{~cd}$ & 0,5 & $2,1 \mathrm{C}$ & 2,5 & 40,0 abc & 45,0 & 88,8 a \\
\hline & 150 & $8,4 \mathrm{c}$ & 9,5 & $20,8 d$ & 23,4 & $0,0 \mathrm{~d}$ & 0,0 & $1,1 \mathrm{~d}$ & 1,3 & 58,5 a & 65,8 & 88,8 a \\
\hline & 180 & $10,4 \mathrm{~b}$ & 11,8 & $21,6 \mathrm{~d}$ & 24,4 & $0,1 \mathrm{~cd}$ & 0,1 & $3,6 \mathrm{~b}$ & 4,0 & $53,1 a b$ & 59,7 & 88,8 a \\
\hline Média & & 10,8 & 12,2 & 31,3 & 35,2 & 1,1 & 1,28 & 3,5 & 3,98 & 42,0 & 47,3 & 88,8 \\
\hline C.V. (\%) & & 6,6 & & 5,3 & & 16,1 & & 9,9 & & 16,9 & & 8,1 \\
\hline \multirow[t]{6}{*}{162} & 30 & $15,1 \mathrm{ab}$ & 12,5 & 52,1 bc & 42,9 & $8,4 \mathrm{a}$ & 6,9 & $10,1 \mathrm{a}$ & 8,4 & $35,6 \mathrm{~b}$ & 29,3 & $121,2 \mathrm{a}$ \\
\hline & 60 & $14,1 \mathrm{~b}$ & 11,6 & $86,5 \mathrm{a}$ & 71,3 & $1,2 \mathrm{c}$ & 1,0 & $4,8 \mathrm{c}$ & 4,0 & $14,7 \mathrm{c}$ & 12,1 & $121,2 \mathrm{a}$ \\
\hline & 90 & $15,3 a b$ & 12,6 & $58,2 \mathrm{~b}$ & 48,0 & $4,0 \mathrm{~b}$ & 3,3 & $8,6 \mathrm{~b}$ & 7,1 & $35,2 a b$ & 29,1 & 121,2 a \\
\hline & 120 & $17,0 \mathrm{a}$ & 14,0 & $59,3 \mathrm{~b}$ & 48,9 & $0,7 \mathrm{c}$ & 0,6 & $2,8 d$ & 2,3 & $41,5 a b$ & 34,2 & 121,2 a \\
\hline & 150 & $14,6 \mathrm{ab}$ & 12,1 & $46,7 \mathrm{~cd}$ & 38,6 & $0,4 \mathrm{c}$ & 0,3 & $2,0 \mathrm{~d}$ & 1,6 & 57,5 a & 47,4 & $121,2 \mathrm{a}$ \\
\hline & 180 & $16,4 a b$ & 13,5 & $41,9 \mathrm{~d}$ & 34,5 & $0,3 \mathrm{c}$ & 0,2 & $5,0 \mathrm{c}$ & 4,1 & $57,7 a$ & 47,6 & $121,2 \mathrm{a}$ \\
\hline Média & & 15,4 & 12,72 & 57,4 & 47,4 & 2,5 & 2,05 & 5,6 & 4,6 & 40,3 & 33,3 & 121,2 \\
\hline C.V. (\%) & & 5,7 & & 4,7 & & 19,2 & & 8,0 & & 15,0 & & 6,6 \\
\hline \multirow[t]{6}{*}{243} & 30 & $16,0 \mathrm{c}$ & 10,5 & $91,4 \mathrm{~b}$ & 60,0 & 17,0 a & 11,2 & 17,0 a & 11,2 & $10,8 \mathrm{~cd}$ & 7,1 & 152,3 a \\
\hline & 60 & $16,2 \mathrm{c}$ & 10,6 & $139,8 \mathrm{a}$ & 91,8 & $1,8 \mathrm{c}$ & 1,2 & $6,0 \mathrm{c}$ & 3,9 & $0,0 \mathrm{~d}$ & 0,0 & $152,3 \mathrm{a}$ \\
\hline & 90 & $32,2 \mathrm{a}$ & 21,1 & $91,4 \mathrm{~b}$ & 60,0 & $5,6 \mathrm{~b}$ & 3,7 & $10,4 d$ & 6,8 & $12,6 \mathrm{c}$ & 8,3 & 152,3 a \\
\hline & 120 & 20,1 bc & 13,2 & $90,8 \mathrm{~b}$ & 59,6 & $0,8 \mathrm{~cd}$ & 0,5 & $3,6 \mathrm{~d}$ & 2,4 & $37,0 \mathrm{~b}$ & 24,3 & $152,3 \mathrm{a}$ \\
\hline & 150 & 20,2 bc & 13,3 & $60,6 \mathrm{c}$ & 39,8 & $0,5 \mathrm{~d}$ & 0,3 & $2,5 d$ & 1,7 & $68,5 a$ & 45,0 & 152,3 a \\
\hline & 180 & $23,7 b$ & 15,5 & $60,6 \mathrm{c}$ & 39,8 & $0,4 d$ & 0,3 & $5,9 \mathrm{c}$ & 3,9 & $61,6 \mathrm{a}$ & 40,5 & $152,3 \mathrm{a}$ \\
\hline Média & & 21,4 & 14,0 & 89,1 & 58,5 & 4,4 & 2,87 & 7,6 & 5,0 & 31,8 & 20,9 & 152,3 \\
\hline C.V. (\%) & & 7,4 & & 2,32 & & 10,07 & & 5,8 & & 13,6 & & 3,9 \\
\hline
\end{tabular}

nd = não detectado. Médias seguidas pela mesma letra, nas colunas, dentro de cada dose, não diferem entre si pelo teste Tukey a $1 \%$. Tr: Trocável, MO: Ligada a matéria orgânica, OxFeA: Óxido de Ferro Amorfo, OxFeC: Óxido de Ferro Cristalino e R: Residual. 


\section{CONCLUSÕES}

1. A adição de doses crescentes de lodo de esgoto aumentou os teores de $\mathrm{Zn}$ extraídos por DTPA; entretanto, de maneira geral, estes teores não aumentaram com o decorrer do tempo de incubação.

2. Houve diminuição da mobilidade de Zn nos solos incubados com lodo, com transferência do elemento ligado à fração matéria orgânica, em sua maior parte, para a fração residual.

3. Para as condições estudadas, a máxima dose de Zn a ser fornecida por aplicação de lodo de esgoto pela legislação americana não promoveu teores fitotóxicos de Zn no solo.

\section{LITE RATURA CITADA}

ANJ OS, A.R.M. \& MATTIAZZO, M.E. Metais pesados em plantas de milho cultivadas em Latossolos repetidamente tratados com biossólido. Sci. Agric., 57:769-776, 2000.

ARAÚJ O, J .C.T. Fracionamento e disponibilidade de zinco em solos incubados com lodo de esgoto. Recife, Universidade Federal Rural de Pernambuco, 2004. 62p. (Tese de Mestrado)

BARROW, N.J . Mechanisms of reaction of zinc with soil and soil components. In: ROBSON, A.D., ed. Zinc in soils and plants. Dordrecht, Kluwer Academic Publishers, 1993. p15-31.

COMPANHIA DE TECNOLOGIA DE SANEAMENTO AMBIENTAL DO ESTADO DE SÃO PAULO - CETESB. Relatório de estabel ecimento de valores orientadores para solos e águas subterrâneas no Estado de São Paulo. São Paulo, CETESB, 2001. 232p.

CHAO, T.T. \& ZHOU, L. Extraction techniques for selective dissolution of amorphous iron oxides from soils and sediments. Soil Sci. Soc. Am. J ., 47:224-232, 1983.

De FILIPPO, B.V. \& RIBEIRO, A.C. Análise química do solo. 2.ed. Viçosa, Universidade Federal de Viçosa, 1997. 26p.

EMPRESA BRASILEIRA DE PESQUISA AGROPECUÁRIA EMBRAPA. Manual de métodos de análise de solo. 2. ed. Rio deJ aneiro, Centro Nacional de Pesquisa de Solos, 1997. $212 p$.

EMPRESA BRASILEIRA DE PESQUISA AGROPECUÁRIA EMBRAPA. Embrapa Solos, Embrapa informática Agropecuária. Manual de análises químicas de solos, plantas e fertilizantes. Brasília, 1999. 370p.

FUENTES, A.; LIORÉNS, M.; SÁEZ, J .; SOLER, A.; AGUIAR, M.I.; ORTUÑO, J.F. \& MESEGUER, V.F. Simple and sequential extractions of heavy metals from different sewage sludges. Chemosphere, 54:1039-1047, 2004.

GOMES, S.B.V. Utilização do lodo de esgoto como fertilizante para cultura do milho: avaliação agronômica e impacto ambiental. Recife, Universidade Federal Rural de Pernambuco, 2004. 59p. (Tese de Mestrado)
HAN, F.X.; BANIN, A.; KINGERY, W.L.; TRIPLETT, G.B.; ZHOU, L.X.; ZHENG, S.J . \& DING, W.X. New approach to studies of heavy metal redistribution in soil. Adv. Environ. Res., 8:113-120, 2003.

HOODA, P.S. \& ALLOWAY, B.J. The plant availability and DTPA extractability of trace metals in sludge-amended soils. Sci. Total Environ., 149:39-51. 1994.

KABATA-PENDIAS, A. \& ADRIANO, D. Trace metal. In: RECHCIGL,J .E., eds. Soil amendments and environmental quality. Flórida, Lewis Publishers,1995. p.139-167.

KIEHL, E.J . Fertilizantes orgânicos. São Paulo, Agronômica Ceres, 1985. 492p.

LINDSAY, W.L. \& NORVELL, W.A. Development of DTPA soil test for zinc, iron, manganese and copper. Soil Sci. Soc. Am. J ., 42:421-428, 1978.

MACHADO, P.L.O.A. \& PAVAN, M.A. Adsorção de zinco por alguns solos do Paraná. R. Bras. Ci. Solo, 11:253-256, 1987.

MCBRIDE, M.B. Toxic metal accumulation from agricultural use of sludge: are USEPA regulations protective? J . Environ. Qual., 24:5-18, 1995.

MCGRATH, S.P.; ZHAO, F.J .; DUNHAM, S.J .; CROSLAND, A.R $\&$ COLEMAN, K. Long-term changes in the extractability and bioavailability of zinc and cadmium after sludge application. J . Environ. Qual., 29:875-883, 2000.

MILLER, W.P.; MARTENS, D.C. \& ZELASNY, L.W. Sequencial extraction procedure for the speciation of particulate trace metal. Anal. Chem., 50:598-601, 1986.

NASCIMENTO, C.W.A.; FONTES, R.L.F.; NEVES, J .C.L. \& MELÍCIO, A.C.F.C. Fracionamento, dessorção e extração química de zinco em Latossolos. R. Bras. Ci. Solo, 26:599606, 2002.

NASCIMENTO, C.W.A.; BARROS, D.A.S.; MELO, E.E.C \& OLIVEIRA, A.B. Alterações químicas em solos e crescimento de milho e feijoeiro após aplicação de lodo de esgoto. R. Bras. Ci. Solo, 28:385-392, 2004.

NASCIMENTO, C.W.A \& FONTES, R.L.F. Correlações entre características de Latossolos e parâmetros de equações de adsorção de cobre e zinco. R. Bras. Ci. Solo, 28:965-971, 2004.

NYAMANGARA, J . \& MZEZEWA, J . The effect of long-term sewage sludge application on $\mathrm{Zn}, \mathrm{Cu}, \mathrm{Ni}$ and $\mathrm{Pb}$ levels in a clay Ioan soil under pasture grass in Zimbabwe. Agric. Ecosys. Environ., 73:199-204, 1999.

OLIVEIRA, F.C \& MATTIAZZO, M.E. Mobilidade de metais pesados em um Latossolo Amarelo distrófico tratado com lodo de esgoto e cultivado com cana-de-açúcar. Sci. Agric., 58:807-812, 2001.

OLIVEIRA, C.; AMARAL SOBRINHO, N.M.B. \& MAZUR, N. Solubilidade de metais pesados em solos tratados com lodo de esgoto enriquecido. R. Bras. Ci. Solo, 27:171-181, 2003.

PARVEEN, Z.; EDWARDS, A.C. \& CRESSER, M.S. Redistribution of zinc from sewage sludge applied to a range of contrasting soils. Sci. Total Environ., 155:161-171, 1994. 
PLANQUART, P.; BONIN, G. PRONE, A. \& MASSIANI, C. Distribution, movement and plant availability of trace metals in soils amended with sewage sludge composts: application to low metal loading. Sci. Total Environ., 241:161-179. 1999.

QIAO, X.L.; LUO, Y.M.; CHRSTIE, P.\& WONG, M.H. Chemical speciation and extractability of $\mathrm{Zn}, \mathrm{Cu}$ and $\mathrm{Cd}$ in two contrasting biosolids-amended clay soil. Chemosphere, 50:823-829, 2003.

SHUMAN, L.M. Fractionation method for soil microelements. Soil Sci., 140:11-22, 1985.

SILVIERA, D.J . \& SOMMERS. L.E. Extractability of cooper, zinc, cadmium and lead in soil. Adv. Environ. Res., 6:47$52,1977$.
STEVENSON, F.J . \& FITCH, A. Chemistry of complexation of metal ions with soil solution organics. In: HUNG, P.M. \& SCHNITZER, M., eds. Interactions of soils minerals with natural organic and microbes. Madison, Soil Science Society America J ournal, 1989. p.29-58.

UNITED STATES - UNITED STATES ENVIRONMENTAL PROTECTION AGENCY - USEPA. Title 40 CFR - Part 503. Final rules: Standards for the use or disposal of sewage sludge. Federal Register, 58:9387-9415, 1993.

UNITED STATES - UNITED STATES ENVIRONMENTAL PROTECTION AGENCY - USEPA. Method 305 2: Microwave assisted acid digestion of siliceous a organically based matrices. Washington, 1996. CD-ROM. 